\title{
DTG-Jahrestagung 2016 in Bonn
}

In dieser Ausgabe berichten wir von einigen Vorträgen der Jahrestagung der DTG 2016 in Bonn. Weitere Berichte folgen in der nächsten Ausgabe.

\section{Plenarvorträge}

Neglected Tropical Diseases

Die erste Keynote Session der Tagung galt den „Neglected Tropical Diseases (NTDs)“. Dr. Adrian Hopkins, Mectizan Donation Programme, Atlanta, gab eine Übersicht über die bisherigen Erfolge gegen die „Chemotherapy Preventable Diseases (PCTs: Oncho, LF, Bilharziose, Trachom, Geohelminthen-Infektionen-STHs)“. Ohne die Bildung von Entwicklungspartnerschaften (PPPs) wäre es nicht dazu gekommen, überhaupt die Eliminierung dieser Infektionskrankheiten als ein realisierbares „Millenium Goal“ zu formulieren. Einer der wichtigsten Schritte zu dieser Entwicklung war die Bereitstellung von Ivermectin (Mectizan $^{\circledR}$ ), für dessen Entwicklung 2015 der Nobelpreis verliehen wurde, durch die Firma Merck Sharp \& Dohme für Onchozerkose und lymphatische Filariose (LF) in Afrika ,for as long as needed“. Es handelt sich um extrem kos- teneffektive Maßnahmen, bei denen durch nur 29 US-Dollar ein DALY (Disability Adjusted life Year) vermieden wird.

Im Jahr 2006 wurden in Afrika für die Eliminierung von Onchozerkose und LF über 300 Mio. Dosen verteilt. Durch die Erfolge

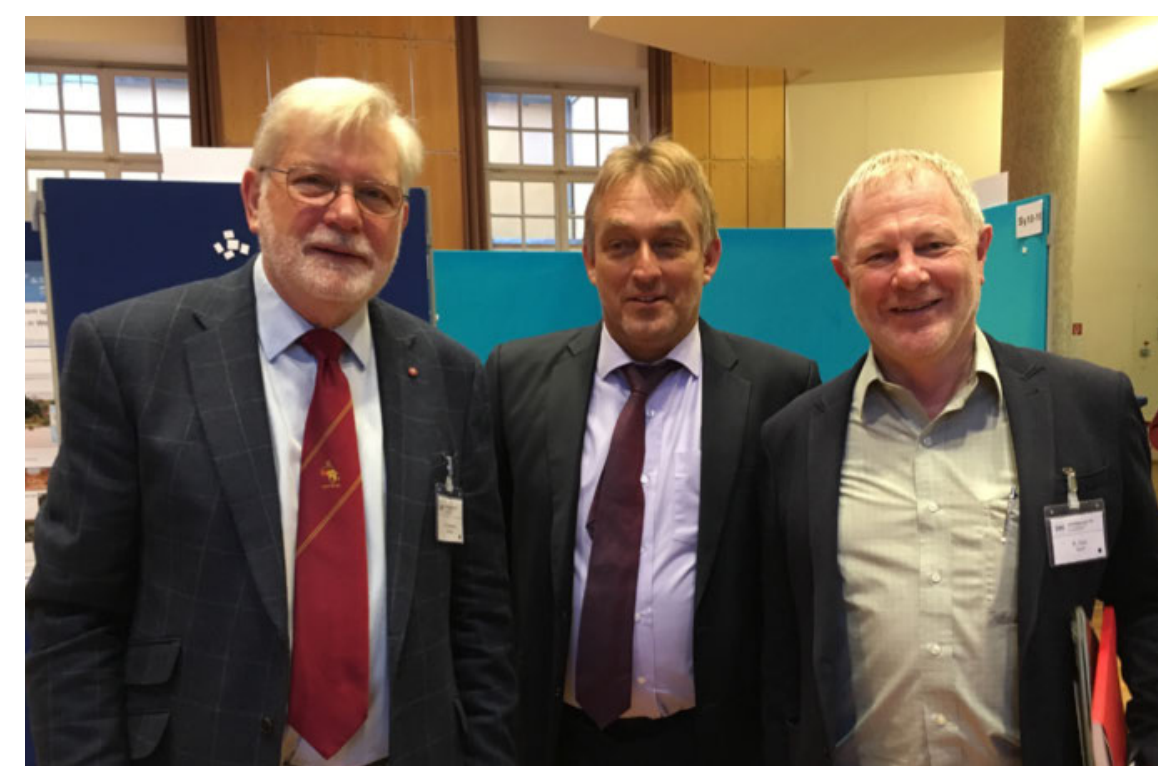

Die beiden Keynote-Sprecher Dr. Adrian Hopkins (links) und Dr. Robert Don (rechts) mit dem Tagungspräsidenten Prof. Achim Hoerauf.

bei der Bekämpfung der LF gehen diese Zahlen (und noch mehr die Behandlungen in Asien mit DEC und Albendazol) bereits wieder zurück, weil immer mehr Länder in die Stufe der Transmissionskontrolle gelangen, die Massenchemotherapie 


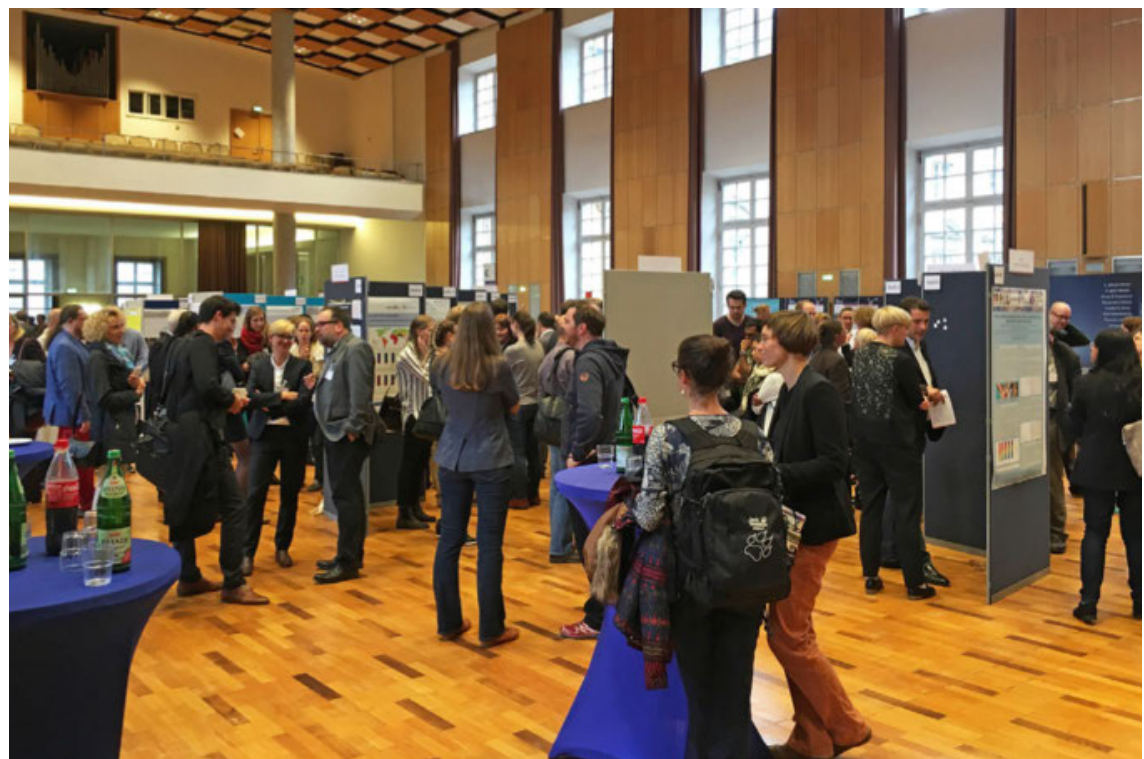

Die Posterausstellung in der großen Aula der Universität Bonn.

(MDA) einstellen und den Erfolg überwachen (sog. TAS, Transmission Assessment Survey, ein WHO-Tool zur Erfolgskontrolle). Die Gefahr ist allerdings, dass durch zu niedrige "Coverage“ ( $<60-70 \%$ der eligible population) die Elimination in etlichen Ländern nicht gelingt, was die Programmdauer erhöhen und zu „Donor fatigue“ führen könnte. Hiervon ist insbesondere die Onchozerkose betroffen, weil mit dem Ende des „African Programme for Onchocerciasis Control“" (APOC) in 2015 keine ähnlich budgetierte Nachfolgeorganisation geschaffen wurde (ESPEN, das „extended special programme for the elimination of neglected tropical diseases", soll mit weniger Budget die 5 chemotherapiepräventablen Infektionen Onchozerkose, LF, Bilharziose, Trachom und STH versorgen). Deshalb werden für die Filarien neue Medikamente benötigt, die nicht nur die Wurmlarven, sondern auch die bis 10 Jahre und länger lebenden Adultwürmer abtöten können.

Neuentwicklungen von Medikamenten gegen NTDs

Auf das Thema der Neuentwicklung von Medikamenten gegen NTDs ging Dr. Robert Don, Head of R\&D bei DNDi, Genf, ein. In den Jahren 1975-1999 entfielen nur $1 \%$ der fast 1400 neu registrierten Medikamente $(n=16)$ auf solche gegen NTDs, weshalb Medecines Sans Frontieres das Geld aus dem Nobelpreis, der 1999 an die Organisation verliehen wurde, zum Aufbau der „Drugs for Neglected Diseases Initiative“, DNDi, verwendete.
DNDi hat sich zum Ziel gesetzt, gegen etliche der NTDs neue Medikamente zu entwickeln, um die Therapie zu vereinfachen beziehungsweise durch praktikable Lösungen (orale Therapie) in entlegenen Gebieten überhaupt erst zu ermöglichen. Über die PDP-Initiative des BMBF fördert auch Deutschland den DNDi, neben anderen großen Donoren wie MSF selbst und der Bill \& Melinda Gates Foundation.

Die derzeitigen Schwerpunkte sind die Entwicklung einer weitgehend oral verfügbaren Therapie gegen Schlafkrankheit (z.B. durch Entwicklung von Fexinidazole in Zusammenarbeit mit Sanofi), geeignete Formulierungen der Medikamente für Kinder mit HIV (ohne Ethanol, erträglicher Geschmack), Zugang zu HCV-Therapie $\mathrm{zu}$ vertretbaren Preisen (Ziel ist 300 US-Dollar pro Therapie), Back-upMedikamente für die Chagas-Krankheit sowie makrofilarizide Therapie gegen Onchozerkose und LF (Zusammenarbeit mit Firmen wie Abbvie, Celgene, Janssen, Merck und präklinischer Entwicklung auch in deutschen Laboren).

Wichtig für den nachhaltigen Erfolg ist aber auch ein stärkeres Engagement der „Emerging Economies“ auf dem afrikanischen Kontinent, in denen schon jetzt doppelt so viele Menschen leben wie in den Least Developed Countries, sowie regulatorische Erleichterungen bei der $\mathrm{Zu}$ lassung. Die EMA ist dafür mit dem Artikel 58 (für Orphan Drugs, die dann nur in Entwicklungsländern zugelassen werden) einen praktikablen Weg gegangen, der hilft, dass die WHO solche Neuzulas- sungen letztlich für die NTDs empfiehlt. Auch die endemischen Länder können sich zusammenschließen, um regulatorische Prozesse gemeinsam durchzuführen oder voneinander zu übernehmen. Diese Maßnahmen werden, vor allem in ihrer Kombination, einen wichtigen Beitrag zu den SDGs der UN leisten.

\section{Neue Erkenntnisse zu MERS-CoV}

Im 2. Plenary Symposium sprach Prof. Christian Drosten, Bonn, über neue Erkenntnisse zum Middle-East-Respiratory-Syndrome-Coronavirus (MERS-CoV), das erstmals 2012 aus einem Patienten in Saudi-Arabien isoliert wurde. Er berichtete, dass retrospektiv bereits aus Material aus dem Jahre 1983 das Virus gefunden wurde. Obwohl alle MERS-CoV sehr miteinander verwandt sind, zeigen die Virussequenzen aus afrikanischen Kamelen, dass das Virus ursprünglich vom afrikanischen Kontinent stammt. Ökologische Untersuchungen zeigten, dass sich das Virus insbesondere in Dromedarkälbern vermehrt und von diesen in großen Mengen ausgeschieden wird. Sie sind die Hauptquelle für die Ansteckung des Menschen. Eine Lebendvakzine für Kamele, die die Ausscheidung des Virus signifikant reduziert, ist kürzlich publiziert worden. Sie basiert auf einem rekombinanten modifizierten Vacciniavirus.

Anders als im Kamel liegt die Reproduktionszahl des MERS-CoV im Menschen deutlich unter 1,0 und die Seroprävalenz in Saudi-Arabien liegt bei nur 0,15\%. Dies zeigt, dass das Virus nicht in der menschlichen Bevölkerung zirkuliert und derzeit kein epidemisches Potenzial besitzt. Allerdings wird es seit Jahrzehnten immer wieder vom Kamel auf den Menschen übertragen, wobei es insbesondere im Rahmen von Krankenhausausbrüchen zu hohen Todesraten kommt. MERS ist also eine klassische Zoonose.

\section{Flüchtlingsmedizin}

Das 3. Plenary Symposium hatte das Problem der Migranten und Flüchtlinge zum Thema. Prof. Wolfgang Eckart, Heidelberg, berichtete aus historischer Perspektive, dass in der deutschen Geschichte immer Migrationsströme zu sozialen und kulturelleren Herausforderungen geführt haben. Die schwierige Assimilation der „Ruhrpolen“ um 1900 und die kriegsbedingten Bevölkerungswanderungen nach 1918 und 1945 waren Situationen, die ohne größere politische Verwerfungen 
bewältigt werden konnten. Danach berichtete Roland Jerusalem, Leiter des Stadtplanungsamts Freiburg, über die Herausforderungen der Unterbringung von Flüchtlingen und Migranten. Er schilderte die in Freiburg gefunden Lösungen, unter anderem stellte er einen Ort der kulturellen Begegnung vor, der auch von Flüchtlingen selbst mitorganisiert wird. Darüber hinaus präsentierte er prämierte Ideen eines Städteplanerwettbewerbs, die bundesweit bereits teilweise umgesetzt sind und sich auch mit der langfristigen Nutzung von Unterkünften beschäftigen wie zum Beispiel spätere Nutzung als Kindertagesstätte - und auch die Verbindung von Arbeitsplätzen und Wohnungen aufgegriffen haben. Anschließend beschrieb Prof. Thomas Junghanss, Heidelberg, eindringlich die medizinischen, sozialen und humanitären Aspekte der in Europa ankommenden Flüchtlinge.

Achim Hoerauf, Beatrix Schumak, Bonn

\section{Junge DTG in Bonn}

Am Freitagnachmittag fand die Veranstaltung ,Junge DTG“ statt. Unter dem Vorsitz von Carsten Köhler und Benno Kreuels, berichteten Sabine Bélard und Sophie Schneitler zum Thema „Tropenmedizin in Deutschland - eine Analyse“ die Ergebnisse ihrer Recherchen zur aktuellen Ausbildungssituation für Tropenmedizin in Deutschland. Hintergrund der Analyse war, dass das Interesse am Fach Tropenmedizin hoch ist, sich dies jedoch nicht in absolvierten Zusatzbezeichnungen Tropenmedizin oder Zertifikaten für Reisemedizin widerspiegelt; die Anzahl der vollständig absolvierten Zusatzweiterbildungen Tropenmedizin zeigte sich in der Bundesärztekammerstatistik zuletzt sogar rückläufig. Der Ausschuss Nachwuchsförderung führte daher bei allen Landesärztekammern eine Umfrage zur Erhebung der Anzahl an Weiterbildungsbefugten für Tropenmedizin, der Bedingungen zur Erlangung der Weiterbildungsbefugnis in Tropenmedizin sowie der Anzahl abgeschlossener Zusatzweiterbildungen durch. Des Weiteren wurden Absolventen der Tropenmedizinkurse in Hamburg und Berlin zu ihrer Motivation den Kurs zu besuchen und ihren weiteren Werdegang befragt. Daten aus dem deutschsprachigen Ausland wurden zum Vergleich eingeholt. Zusammengefasst zeigt sich, dass eine Ausbildung in Tropenmedizin nicht flächendeckend in Deutschland möglich ist und dass die Voraussetzungen zur Erlan- gung der Weiterbildungsbefugnis in Tropenmedizin zwischen den Bundesländern stark variieren. Die Umfrage unter den Tropenkursabsolventen zeigt, dass trotz initial hohem Interesse am Fachgebiet und an der Erlangung der Zusatzbezeichnung Tropenmedizin nur wenige Kollegen die Zusatzweiterbildung abschließen; die beschränkten Ausbildungsmöglichkeiten in Deutschland wurden häufig als Grund hierfür genannt. Im deutschsprachigen Ausland ist Tropenmedizin ein Facharzt oder Teil eines Facharztes. Die erhobenen Daten bieten dem Ausschuss Nachwuchsförderung nun die Grundlage für die Erarbeitung von Vorschlägen für eine Verbesserung der Ausbildungssituation in Tropenmedizin in Deutschland.

Danach fand ein spannender Science Slam zum Thema „Tropische Hitze - Fall und Forschung“ statt. Acht junge Kolleginnen und Kollegen präsentierten in Kurzvorträgen außergewöhnliche klinische Fälle oder spannende Forschungsergebnisse. Das Publikum der sehr gut besuchten Veranstaltung war danach gefragt, über die beste Präsentation abzustimmen. Tanja Peters aus Hamburg durfte sich als glückliche Gewinnerin freuen. Carsten Köhler, Tübingen, Sophie Schneitler, Leipzig, Sabine Bélard, Berlin

\section{Session der GTP}

Die Session der Gesellschaft für Tropenpädiatrie und Internationale Kindergesundheit (GTP) e.V. war ein konstruktiver forschungsorientierter Austausch: Teilnehmer und Vortragende haben sich vornehmlich den Themen der Verbesserung der Kindergesundheit in ressourcenarmen Ländern beziehungsweise der Qualität der präventiven und kurativen pädiatrischen Versorgung gewidmet. Dabei wurden zum einen seltene infektiologische Fälle betrachtet: Cancrum-oris-Fälle in Tansania durch den GTP-Vorsitzenden Carsten Krüger, der Fall einer Strongyloides-stercoralis-Infektion bei einer immunsupprimierten Patientin durch Martin Dennebaum sowie Komplikationen des Läuserückfallfiebers durch Christa Kitz. Und zum anderen wurde die Bedeutung neonatologischer Versorgung dargestellt, darunter: die neonatale Sterblichkeitsraten in Kamerun durch Bena-Boupda, die erfolgreiche Behandlung eines Neonaten mit Ebola durch Jenny Dörnemann sowie die Bedeutung von Tanzen und Singen in der psychomotorischen Entwicklung von Kindern durch J. Richter.

Schließlich gab es noch sehr spannenden infektionsepidemiologische Vorträge zum Thema der MDR-TBC bei Migranten (Schulze-Sturm), die Entwicklung einer Multiplex-PCR-Diagnostik zur Erfassung von febrilen, malariaähnlichen arboviralen Erkrankungen in der Lake-ViktoriaRegion durch P. Koliopoulos und das Screening für extrapulmonale TBC-Manifestationen mittels eines standardisierten Ultraschallverfahrens in Indien durch S. Weber.

Die Bandbreite der Themen dokumentiert die beeindruckenden Aktivitäten im Bereich der Tropenpädiatrie, die ganz offensichtlich auf sehr großes Interesse stoßen - der Seminarraum war übervoll.

Stephan Gehring, Mainz

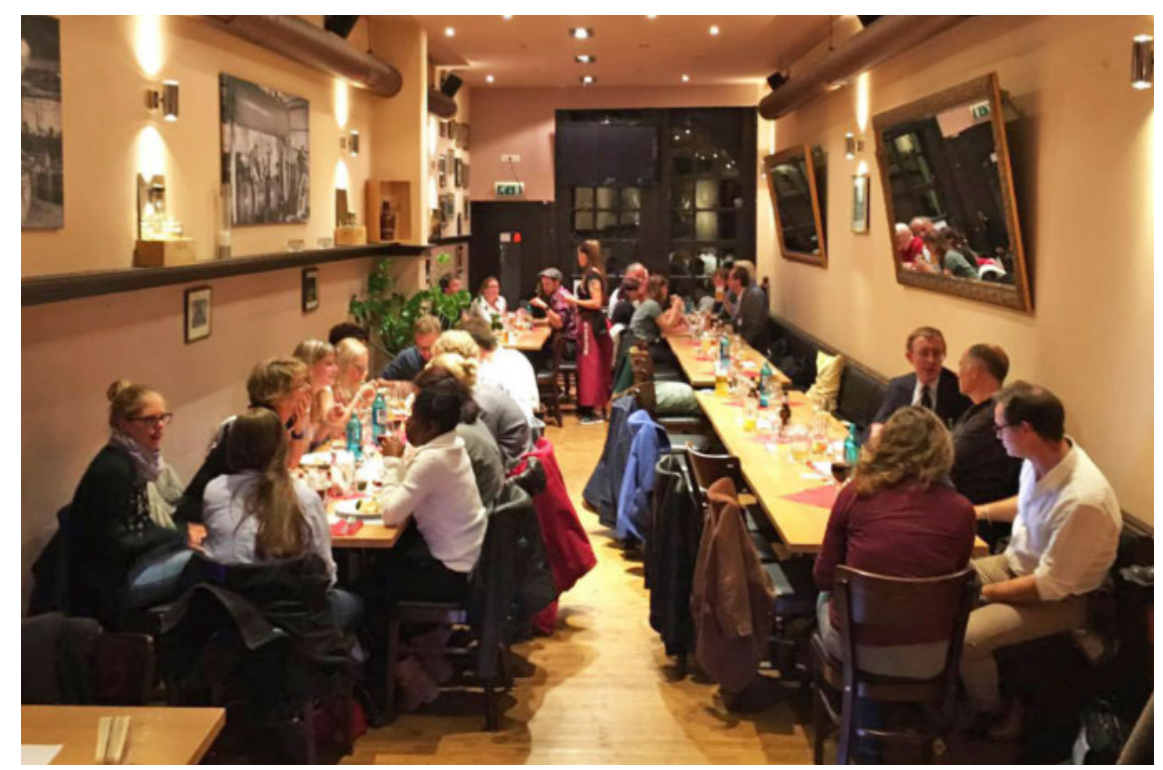

Gesellschaftsabend der DTG im Machold in der Bonner Altstadt. 